\title{
Correction to: Fracture patterns of the hyoid-larynx complex after fatal trauma on the neck: retrospective radiological postmortem analysis of 284 cases
}

\author{
Henri M. de Bakker ${ }^{1}$ (D) Moritz V. Warmbrunn ${ }^{2}$ - Peggy van den Biggelaar ${ }^{2} \cdot$ Vidija Soerdjbalie-Maikoe $^{3}$ (D) \\ Bernadette S. de Bakker ${ }^{2}$ (D)
}

Published online: 1 September 2020

(C) Springer-Verlag GmbH Germany, part of Springer Nature 2020

\begin{abstract}
Purpose Fatal trauma on the neck occurs frequent in forensic cases and often results in fractures of the hyoid-larynx complex. The aim of the present study is to provide an overview of fractures in the hyoid-larynx complex that occur due to fatal trauma on the neck and can be observed by radiological evaluation.

Methods Radiological images from a forensic radiological database created in the Groene Hart Hospital, Gouda, the Netherlands were used for analysis. Hyoid-larynx complexes were explanted in 284 individuals who accordingly to the forensic pathologist allegedly died from fatal trauma on the neck. These explants were imaged with conventional X-rays in eight directions and a CT scan. Radiological images were analyzed for fractures, dislocations, joints, and anatomical variations by a trained analyst and a radiologist.

Results In 281/284 cases, the hyoid bone and, in 252/284 cases, the thyroid cartilage could be assessed. In 56 victims (20\%), the hyoid bone was fractured, 55 times in the greater horn, 1 fracture in the body. The calcified superior horn of the thyroid showed a fracture in 101 victims (40\%). The calcified cricoid cartilage was fractured in one case. Multiple fractures were found in 31/284 cases $(11 \%)$. Joints between the greater horn and body of the hyoid were present in $74 \%$.
\end{abstract}

\section{Key points}

1. Thyroid cartilage superior horn fractures are most frequently found in fatal trauma on the neck.

2. Hyoid bone fractures most frequently occur in the greater horns.

3. Joints between the greater horn and the body of the hyoid bone were found in $74 \%$ of 281 cases.

4. Dislocations of hyoid joints are rare in fatal trauma on the neck victims $(2,5 \%)$.

Henri M. de Bakker and Moritz V. Warmbrunn are shared first authors

Some text parts of the published article https://doi.org/10.1007/s00414019-02241-8 contain uncorrected wording (placeholders). To avoid any misinterpretation, the whole article is published below in full with the corrected data

The online version of the original article can be found at https://doi.org/ $10.1007 / \mathrm{s} 00414-019-02241-8$

Electronic supplementary material The online version of this article (https://doi.org/10.1007/s00414-020-02382-1) contains supplementary material, which is available to authorized users.

Bernadette S. de Bakker

b.s.debakker@amsterdamumc.nl

1 Department of Radiology, Groene Hart Hospital, Bleulandweg 10, 2803 HH Gouda, Netherlands
2 Department of Medical Biology, Section Clinical Anatomy \& Embryology, Amsterdam UMC, University of Amsterdam, Meibergdreef 9, 1105 AZ Amsterdam, Netherlands

3 Division of Special Services, Section Forensic Pathology, Netherlands Forensic Institute, P.O. Box 24044, 2490 AA The Hague, Netherlands 
Conclusion Trauma on the neck leads most frequently to fractures of the superior horn of the thyroid cartilage and second most to fractures in the greater horn of the hyoid bone. (Forensic) radiologists should be aware of uncommon fracture locations, anatomical variations, and dislocations in the hyoid-larynx complex.

Keywords Hyoid-larynx complex $\cdot$ Forensic radiology $\cdot$ Hyoid fracture $\cdot$ Thyroid fracture $\cdot$ Postmortem computed tomography

\author{
Abbreviations \\ CT Computed tomography \\ GH Greater horn \\ GHH Groene Hart Hospital \\ HLC Hyoid-larynx complex \\ MRI Magnetic resonance imaging \\ NFI Netherlands Forensic Institute \\ TC Thyroid cartilage \\ TBCT Total body computed tomography
}

\section{Introduction}

The hyoid-larynx complex (HLC) is of significant importance for forensic death cases when fatal trauma on the neck is considered as cause of death and homicides associated with fatal trauma on the neck occur relatively frequent $[1,2]$. Thirteen percent of all homicides in suburbs of Paris and 20.9-22.9\% of homicides in two Scandinavian capitals were caused by asphyxia, including manual and ligature strangulation, smothering, choking, drowning, or a combination of different methods [3, 4]. If a body is examined relatively soon after death, cues for homicidal asphyxia, e.g., petechiae of the conjunctivae and/or sclerae, can give an indication for the cause of death, as they occur in, respectively, $89 \%$ and $86 \%$ of manual and ligature strangulation cases [5]. However, circumstances are not always unambiguous. To interpret forensic evidence, profound knowledge about normal and aberrant anatomy is necessary. If indicated, the pathologist can decide to explant the HLC for additional radiological examination [6]. Radiological imaging is already being used for nonfatal causes of hyoid fracture in cases of vomiting, hyperextension, trauma against the neck during fight sports, traffic accidents, and iatrogenic damage after neck surgery [7-12]. Forensic radiology is gaining popularity; postmortem CT and MRI scans are becoming standard procedure for legal death investigations in an increasing number of institutions [13-17]. In recent studies on strangulation, postmortem $\mathrm{CT}$ is superior to autopsy for detecting, e.g., the gas bubble sign or fracture depiction [14]. Most authors describe case studies or small retrospective studies; therefore, the need for large retrospective studies is rising. The aim of the present study is to describe typical fracture locations in 284 explanted HLC's with suspected fatal trauma on the neck, by retrospective radiologic postmortem analyses.

\section{Fatal pressure on the neck}

There are multiple ways in which pressure on the neck can become fatal. First, pressure on the neck gives compression of arteries and veins. The latter occurs the fastest as some veins are located directly under the skin. Occlusion of neck veins leads to characteristic findings such as edema, cyanosis, or petechiae cranial from the neck. Occlusion due to compression of carotid arteries in the neck occurs at around $3.5 \mathrm{~kg}$ of pressure [18]. Second, intermittent occlusion can lead to carotid artery dissection, and persistent occlusion of the carotid circulation leads to irreversible brain damage if the occlusion persists for $4 \mathrm{~min}$ or longer [6,19]. Third, if pressure is applied on baroreceptors in the carotid sinuses, acute bradycardia can occur which can be enhanced by a range of drugs potentially leading to cardiac arrest [20]. Fourth, compression of the airway can result in lifting of the larynx which can lead to closing of the pharynx by the base of the tongue or to airway occlusion. As the force needed for airway occlusion is around $16 \mathrm{~kg}$, another mechanism which needs less force can occur more frequently such as occlusion of arteries and veins [21].

\section{The hyoid-larynx complex}

To evaluate the HLC, the following structures can be identified, most cranially the stylohyoid process of the temporal bone of the skull which is connected to the hyoid bone by the stylohyoid ligament. The hyoid bone is composed of a body with two lesser horns on the cranial part of the body and two greater horns that elongate the body bilaterally. The thyroid cartilage has two superior horns pointing cranially on both lateral sides and two smaller inferior horns pointing in the opposite direction on the caudal end of the cartilage (Fig. 1). The hyoid bone and thyroid cartilage are connected by the thyrohyoid membrane, which contains three stronger parts: one medial and two lateral thyrohyoid ligaments. More caudally, the cricoid cartilage, which is the only circular cartilage around the airway, articulates with the inferior horns of the thyroid cartilage by cricothyroid joint facets. The arytenoid cartilages articulate with the cranial arytenoid joint facets of the cricoid cartilage.

In humans, the HLC shows a broad morphological variety in relation to its embryonic development [22, 23]. Many differences have been described due to sex, race, age, and differences in body proportions [1, 24-27]. Some anatomical variations can even mimic fractures. For example, non-fusion 

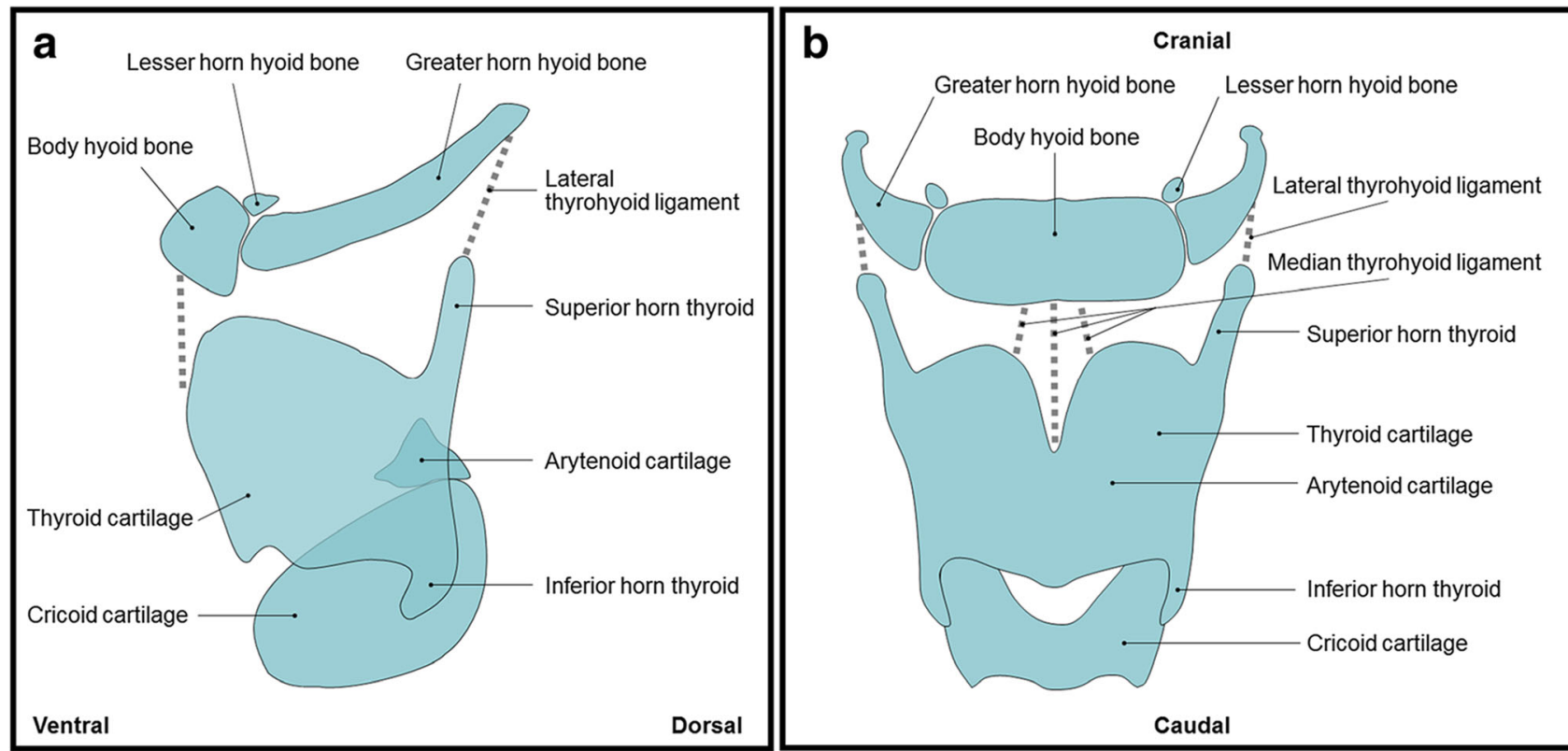

Fig. 1 Anatomy of the hyoid-larynx complex a Lateral view of the hyoid-larynx complex. b Ventral view of the hyoid-larynx complex

between the superior horn of the thyroid and thyroid body could incorrectly be identified as a fracture [23].

\section{Material and methods}

\section{Dutch forensic asphyxia protocol}

Most forensic autopsies in the Netherlands take place at the Netherlands Forensic Institute (NFI), The Hague,
The Netherlands. Since 2000, the NFI set up a collaboration with the radiology department at the Groene Hart Hospital (GHH) in Gouda, the Netherlands [28] to perform radiologic examination prior to forensic autopsy. Nowadays, the total body computed tomography (TBCT) has become standard practice. The current workflow (Fig. 2) is that after the TBCT and forensic autopsy, the pathologist removes the HLC (explant), which contains the hyoid bone, thyroid bone, and direct surrounding structures such as ligaments and muscles, when there is suspicion of trauma on the neck. The

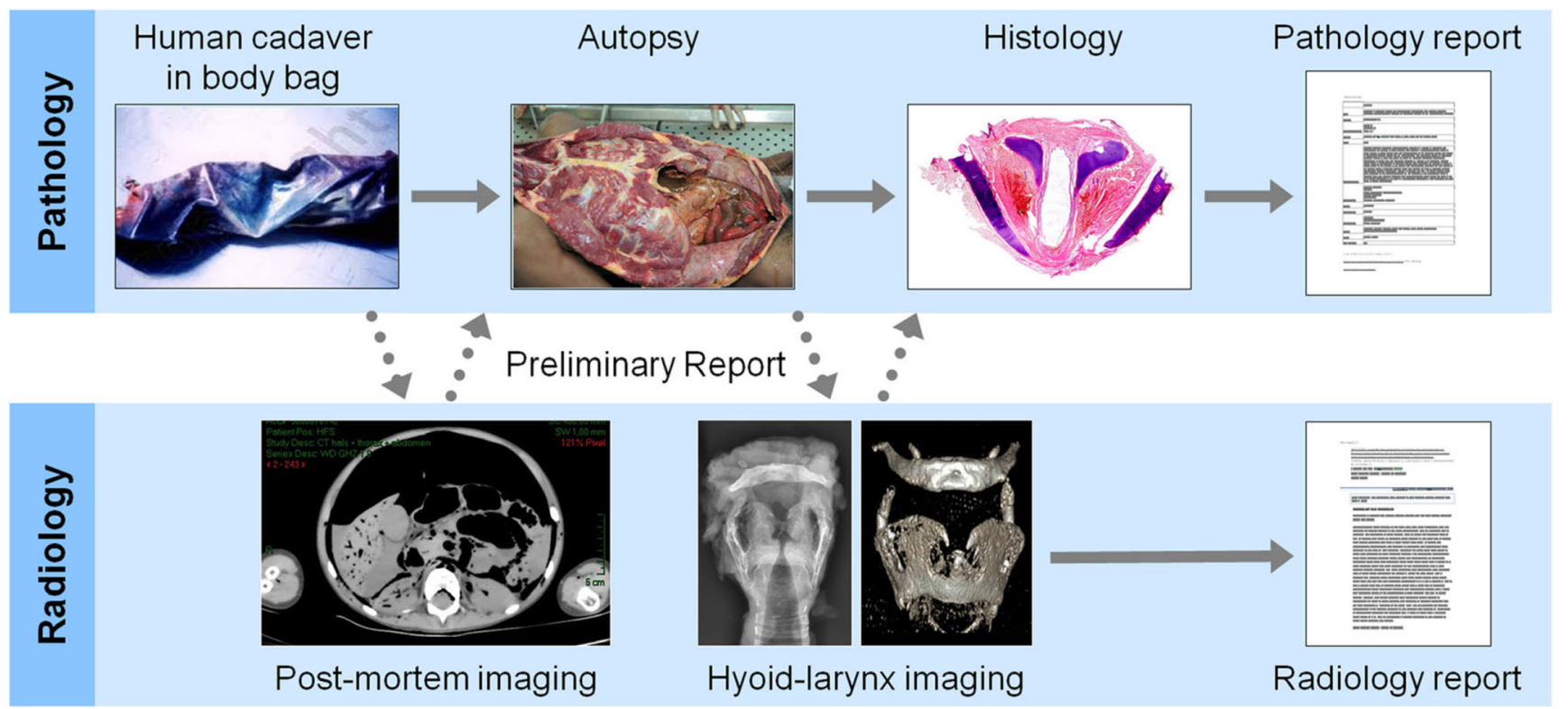

Fig. 2 Overview of hyoid-larynx complex research protocol after fatal trauma on the neck: After fatal trauma led to death, the first step is postmortal imaging. A total body CT is performed, followed by a forensic autopsy. The explanted hyoid-larynx complex is again offered to the radiology department, imaged by conventional X-rays and CT scan, and is subsequently histologically analyzed. Based on this, the radiology and pathology reports are created 
explant is sent back to the GHH for further radiological examination. First, conventional X-ray images are obtained in eight different directions. Second, a CT scan of the explant is performed, with three-dimensional (3D) reconstructions (Fig. 2).

\section{Forensic database}

All forensic cases presented by the NFI to the GHH since 2000 were radiologically analyzed. Subsequently, a forensic pathologist investigated the cause of death [28]. This way, a forensic-radiological database currently containing over 2300 cases was established. We retrospectively studied 284 HLCs of persons that died of allegedly asphyxia by trauma on the neck in the period between 2000 and 2013.

\section{Image acquisition}

Three different techniques were used for image acquisition: in the first years, the conventional X-rays of the explant were made on the mammography system. Since 2005, we use the digital x-room (Carestream X-ray scanner; Carestream 7500 and later Carestream DRX Evolution, Carestream Health Netherlands B.V., Eemnes, Netherlands). Conventional Xray images were obtained in eight different directions: AP, left lateral, right lateral, cranio-caudal, left oblique 30 and $60^{\circ}$, and right oblique 30 and $60^{\circ}$, with protocol parameters, $42 \mathrm{kV}$, $10 \mathrm{mAs}$, FF $110 \mathrm{~cm}$, small focus, and no grid. In addition to conventional X-rays, a CT of the explanted HLC was obtained: in the first years of the study with a Siemens Somatom 4 (Siemens, Erlangen, Germany) and, since 2009, a Toshiba Aquilion 32 slice and a Toshiba Aquilion 64 slice (Toshiba Medical Systems Europe B.V., Zoetermeer, Netherlands), protocol parameters $80 \mathrm{kV}, 150 \mathrm{mAs}, 0,5 \mathrm{~s}$ rotation time, soft tissue, and bone window, 3D reconstructions were also acquired (Fig. 2).

\section{Radiological assessment}

The hyoid bone follows the normal fracture characteristics of bone in general. A fracture line in the greater horn, dislocation and/or angulation can be seen. The cartilage of the thyroid needs a certain degree of calcification to be able to assess. Specific in the superior horn, an interruption line can be seen whether or not in combination with dislocation and/or angulation. When subtle, it may be missed on CT when it parallels the transversal scan plane.

\section{Data analysis}

All 284 cases were retrospectively reanalyzed by a trained analyst together with an experienced forensic radiologist (Fig. 2). Fractures and luxations were identified on the series
Table. 1 HLC fracture patterns after fatal trauma on the neck

\begin{tabular}{|c|c|c|}
\hline Fractured hyoid bone & Cases & Percentage $(\%)^{*}$ \\
\hline Hyoid body & 1 & 0.4 \\
\hline Greater horn fractured & 55 & 19.6 \\
\hline (Left GH) & $(22)$ & $(7.8)$ \\
\hline (Right GH) & (18) & (6.4) \\
\hline (GH left/right**) & (3) & $(1.1)$ \\
\hline (GH bilateral) & (12) & (4.3) \\
\hline No fracture hyoid bone & 225 & 80.0 \\
\hline Hyoid data without missing data & 281 & 100 \\
\hline Hyoid missing data & 3 & - \\
\hline HLC data total & 284 & - \\
\hline Fractured thyroid cartilage (TC) & Cases & Percentage $(\%)^{*}$ \\
\hline \multicolumn{3}{|l|}{ Superior horn $(\mathrm{SH})$} \\
\hline $\mathrm{SH}$ fractured & 101 & 40.1 \\
\hline (Left SH) & (27) & $(10.7)$ \\
\hline (Right SH) & (29) & $(11.5)$ \\
\hline (Bilateral SH) & (45) & $(17.9)$ \\
\hline No fracture $\mathrm{SH}$ & 151 & 59.9 \\
\hline TC data without missing data & 252 & 100 \\
\hline TC missing data & 32 & - \\
\hline HLC data total & 284 & - \\
\hline Combined fractures & Cases & Percentage $(\%)$ \\
\hline GH and superior horn & 22 & 7.7 \\
\hline $\mathrm{GH}$ and miscellaneous & 5 & 1.8 \\
\hline Superior horn and miscellaneous & 4 & 1.4 \\
\hline Total combined fractures & 31 & 10.9 \\
\hline HLC data total & 284 & 100 \\
\hline Other fracture locations & Cases & Percentage $(\%)^{*}$ \\
\hline Anterior thyroid (paramedian) & 14 & 4.9 \\
\hline Right inferior horn TC & 1 & 0.35 \\
\hline Cricoid & 1 & 0.35 \\
\hline Total number miscellaneous fractures & 16 & 5.6 \\
\hline HLC data total & 284 & 100 \\
\hline
\end{tabular}

The table shows the absolute and relative number of isolated and combined fractures of the hyoid-larynx complex. Percentages are based on the available data, not every radiologic image allowed evaluation of certain structures such as the thyroid cartilage. *Percentages were calculated without missing data. **If only the explanted hyoid bone was available for radiologic evaluation, discrimination between the left and right side was not possible

of 8 conventional X-rays and CT scan of the explanted HLC including $3 \mathrm{D}$ reconstruction. HLCs were not always completely available for radiologic evaluation, e.g., when the hyoid bone or the thyroid was missing after excavation of a body or when the thyroid was not calcified, cases were labeled as missing data. Percentages were calculated without missing data (Tables 1 and 2).

\section{Results}

\section{Data characteristics}

The mean age of included subjects was 45 years with a range from 1 to 98 years; male female ratio was 1:1.1. Radiological analysis of 284 HLCs led to the following results. 
Table 2 Joints and dislocations in the hyoid bone after fatal trauma on the neck

\begin{tabular}{lcc}
\hline Greater horn-hyoid body joints & Cases & Percentage $(\%)^{*}$ \\
\hline Joint bilateral & 172 & 61.2 \\
Joint unilateral & 35 & 12.5 \\
$\quad$ (Left) & $(12)$ & $(4.2)$ \\
$\quad$ Right) & $(19)$ & $(6.9)$ \\
$\quad$ Left or right**) & $(4)$ & $(1.4)$ \\
No joint & 74 & 26.3 \\
Hyoid data without missing data & 281 & 100 \\
Hyoid missing data & 3 & - \\
HLC data total & 284 & - \\
& & \\
Dislocations & Cases & Percentage $(\%)^{*}$ \\
Unilateral & 7 & 2.5 \\
Bilateral & 0 & - \\
No dislocation & 274 & 97.5 \\
Hyoid data without missing data & 281 & 100 \\
Hyoid missing data & 3 & - \\
HLC data total & 284 & - \\
\hline
\end{tabular}

Description of the number of cases and percentages of joints observed between the greater horn and body of the hyoid bone and dislocations in this joint after fatal trauma on the neck. Data are based on radiological evaluation. *Percentages were calculated without missing. **If only the explanted hyoid bone was available for radiologic evaluation, discrimination between the left and right side was uncertain

\section{Fractures}

From the 284 HLCs concerning persons that died allegedly by trauma on the neck, 281 hyoid bones ( 3 missing) and 252 sufficiently calcified thyroids ( 32 missing; 26 not present, and 6 insufficiently calcified) could be radiologically examined. Fifty-six out of 281 hyoid bones (20\%) were fractured. In 55 cases, fractures were located on the greater horn of the hyoid bone. In one case, the body of the hyoid was fractured. The 55 cases comprised 12 bilateral fractures, 18 fractures of the right greater horn, and 22 fractures of the left greater horn. In the three cases where only the hyoid was present, the thyroid as reference point was missing. In these cases, the side of the fractured horn could not be determined on the X-ray (Table 1; Figs. 3, 4 and 5). The calcified superior horn of the thyroid revealed fractures in 101 out of 252 cases $(40.1 \%)$, including 45 bilateral fractures, 27 left-sided fractures, and 29 right-sided fractures (Table 1; Figs. 3 and 4). The calcified cricoid cartilage was fractured in one case (on the right side).

\section{Combined fractures}

In 31 out of 284 cases (10.9\%), multiple fractures were observed. In 22 cases, a combination of fractures in the greater horn of the hyoid and the superior horn of the thyroid cartilage was seen. Five cases revealed a fracture of the greater horn in combination with other fractures than the superior horn of the thyroid cartilage. In four cases, the superior horn of the thyroid cartilage was fractured together with another structure of the HLC (other than the greater horn of the hyoid bone) (Table 1).

\section{Dislocations of joints}

In 207 out of 281 hyoid bones (73\%) joints were observed between the greater horn and the body of the hyoid (GHBH). In 35 specimens, joints were present unilaterally and in 172 cases bilaterally. In 74 cases no joints were observed in the hyoid bone. Unilateral dislocation of the greater horn was observed in seven cases $(2.5 \%)$ out of 281 (Table 2).
Fig. 3 Overview of fractures of the hyoid-larynx complex after fatal trauma on the neck. The most frequent fracture of the hyoid-larynx complex was observed in the superior horn of the thyroid. The second most frequent fractures were observed in the greater horn of the hyoid bone. Rare occurrences were fractures in the lesser horn of the hyoid bone, hyoid bone body, inferior horn of the thyroid, and cricoid. $\#$ = fracture

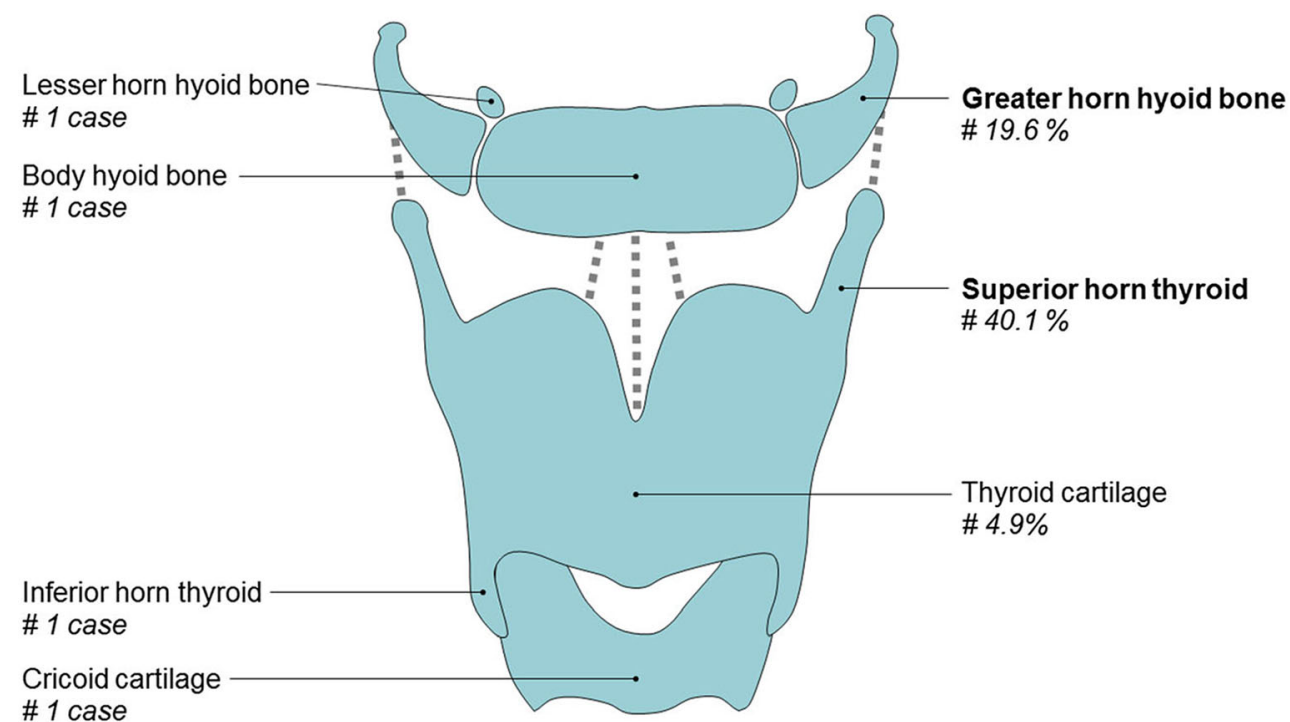


Fig. 4 X-ray view of the hyoidlarynx complex in three cases with fatal trauma on the neck. a Case 1, anteroposterior projection shows fracture of the right greater horn of the hyoid bone and assumed fracture of the right superior horn of the thyroid (arrows). b Same case as in A, oblique projection, with better visualization of both fractures on the right (arrows). c Case 2, lateral projection shows fracture of right superior horn thyroid (arrow). d Case 3, oblique projection, detail, with fracture of the right superior horn of the thyroid (arrow)
Fig. 5 X-ray views of hyoid bone with fractures after fatal trauma on the neck. a Fracture of the greater horn on both sides with minimal dislocation (arrows). Bilateral fusion between hyoid bone body and greater horn. $\mathbf{b}$ Dislocation between the greater horn and body of the hyoid bone on the right side and a fracture halfway the greater horn on the opposite site (arrows). c Fracture of the greater horn on both sides with dislocation and medial angulation (arrows). d Fracture of the greater horn on the left (arrow) and a visible joint apparent between the greater horn and hyoid body bilaterally
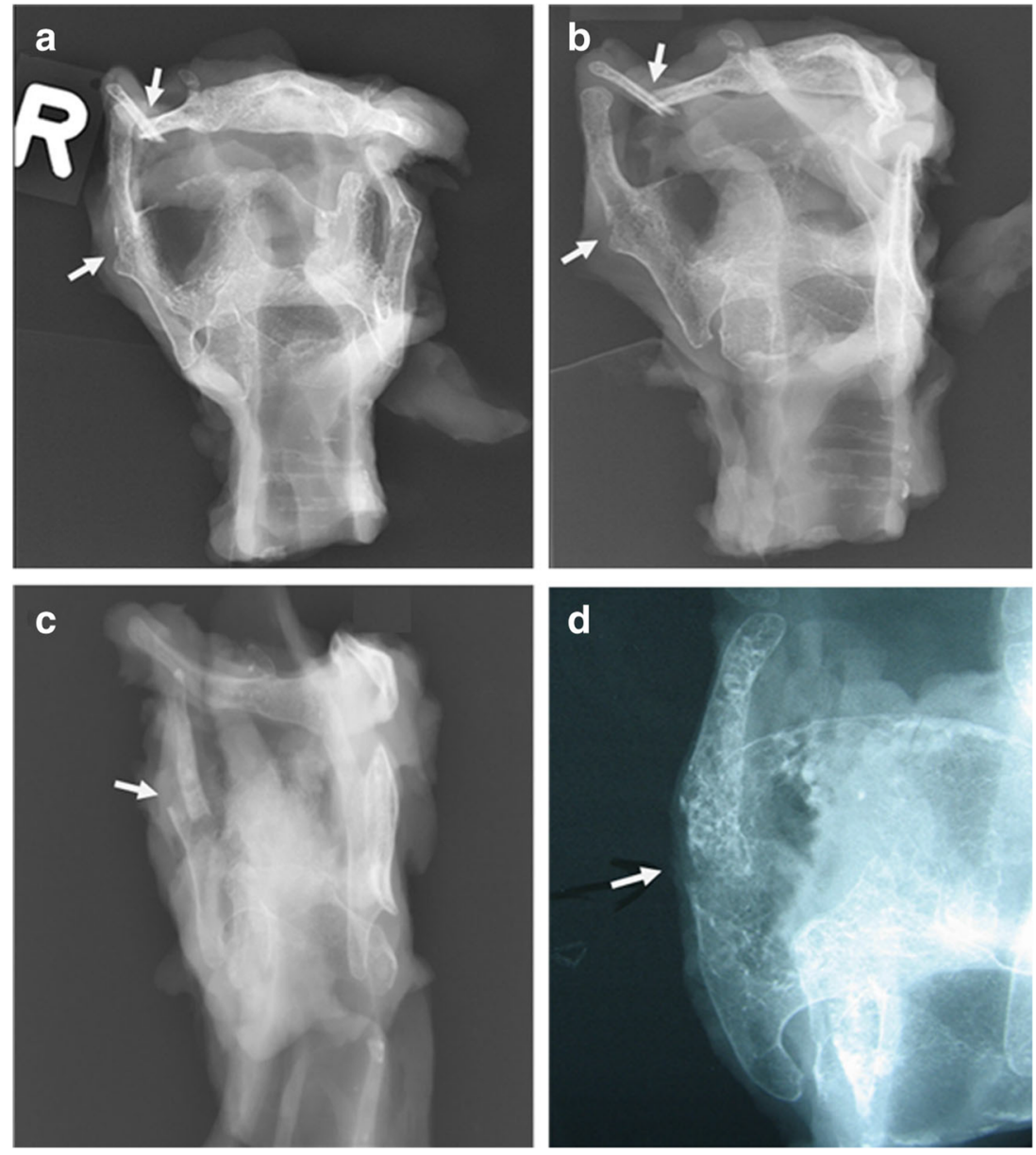
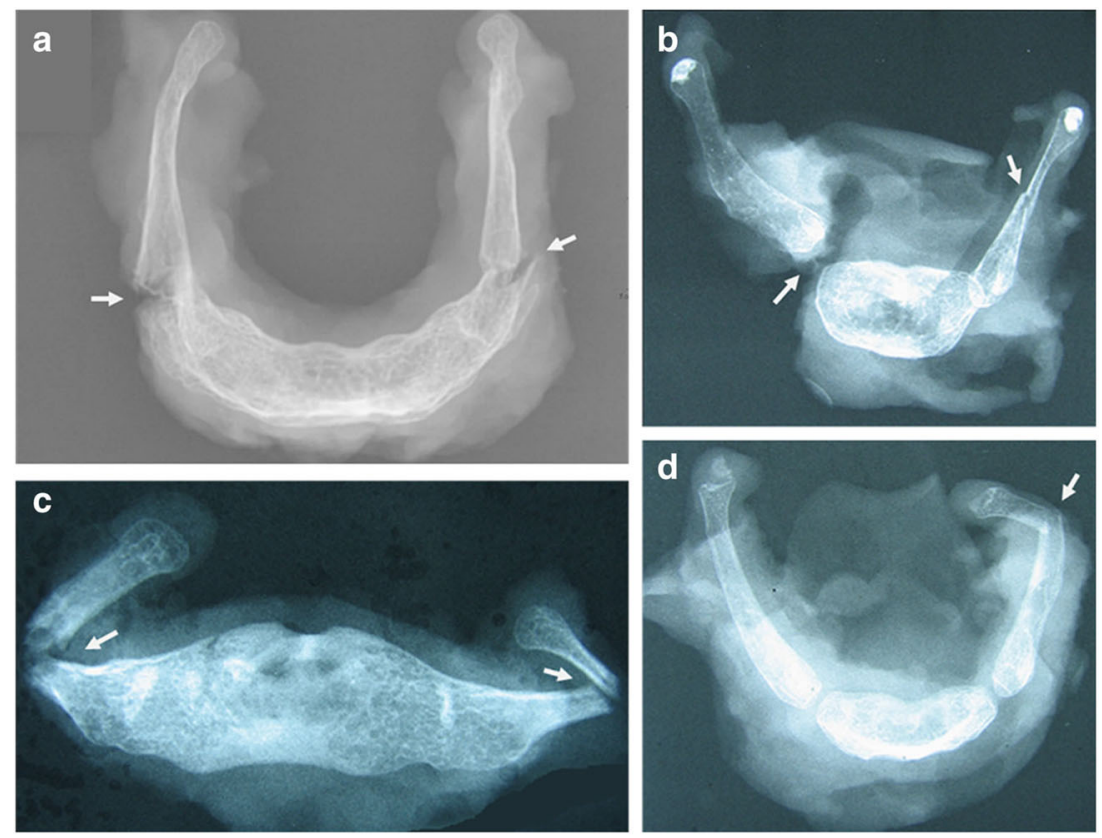


\section{Anatomical variations}

In $63 \%$ of the 284 cases, anatomical variants were observed. In one special variant case, the greater horn and lesser horn were of similar length and both were fractured (Fig. 6). The anatomical variations are very diverse and do not fit in the context of this manuscript, so we do not take it further into consideration.

Summarizing, in this radiological study, we found that fatal trauma on the neck showed most frequently fractures in the superior horn of the calcified thyroid cartilage. The greater horn of the hyoid bone was fractured second mostly. The thyroid body was fractured in a low number of cases $(4.9 \%)$. About $11 \%$ of the cases showed a combination of fractures. Rare fractures were also found, for example, solitary fractures of the lesser horn, the body of the hyoid bone, the calcified cricoid, as well as the inferior horn of the thyroid cartilage (Fig. 3).

\section{Discussion}

\section{Risk factors for fractures}

Factors that contribute to hyoid fracture have been a controversial subject of discussion in the literature. It has been proposed that fusion of the greater horns with the body increases the risk of hyoid fracture [29-31]. The mean age for unilateral or bilateral fusion in males and females is debated. After 60 years, all hyoid bones were fused in an Indian postmortem study, while in another Indian study, non-fusion of the hyoid bone was also seen in the age range between 60 and 70 years $[29,30]$. In our study, we observed fractures of the greater horn even though joints were present between the greater horn and hyoid body (Fig. 5b, d). Some studies implicate that both age and gender influence the risk of hyoid fractures [1, 32, 33]. However, others propose that not the general variables such as sex determine the location of a fracture, as angles and curvature of the hyoid bone can vary broadly, but rather anatomical variations as asymmetry of the hyoid bone [34].

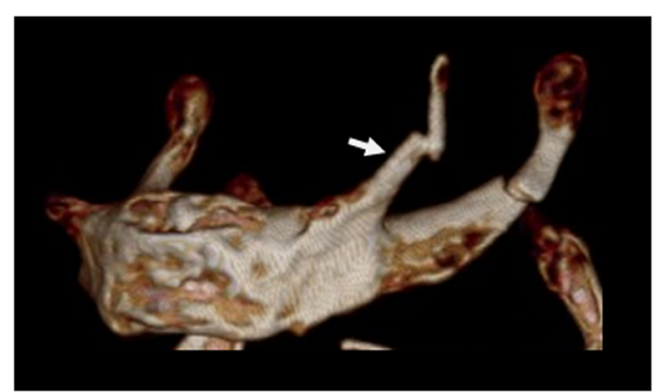

Fig. 6 Example of a rare fracture combination in the hyoid bone after fatal trauma on the neck. The lesser horn on the left (white arrow) has, as a variant, almost the same length as the left greater horn of the hyoid bone on this 3D CT image. Both left horns are fractured after fatal trauma on the neck in this specimen, from a 46-year-old male
Anatomical variations and nonfatal pressure on the neck

To correctly interpret postmortem radiological images, knowledge of normal anatomy and anatomical variations of the HLC is important as some anatomic variations can mimic fractures. In $63 \%$ of the 284 cases, anatomical variants were observed. In one case, the greater horn and lesser horn were of almost similar length due to an anatomical variant in which the left lesser horn was elongated. Interestingly, the greater horn and lesser horn were fractured in the same specimen (Fig. 6). It should also be considered that intense calcifications are not limited to older individuals. Calcification of the triticeal cartilage, which is located in the thyrohyoid ligament, can falsely appear as an avulsion fracture of the superior horn of the thyroid cartilage $[35,36]$. The use of X-rays in different directions can help distinguish between fractures and inconsistently calcified cartilage [37].

Nonfatal hyoid bone fractures are a rare entity as the general prevalence of hyoid fractures is mentioned as low as $0.002 \%$ of all fractures by several researchers $[2,12$, $33,38]$. However, this number is first described in 1942 and may be not representative in modern societies [39]. Nevertheless, in the period from 1954 to 2003, only 31 cases of the hyoid bone fractures were described due to nonfatal pressure on the neck [33]. If hyoid fractures occur due to nonfatal pressure on the neck, usually symptoms as pain in the neck or throat, dysphagia, dyspnea, wheezing, coughing, pain during speaking, or in one case only a clicking sensation in the throat were described [7-12]. As these fractures are so rare, a hyoid fracture found in a radiological postmortem evaluation in potential homicidal circumstances should raise suspicion of third-party involvement and therefore needs to be thoroughly analyzed for signs of fatal pressure on the neck. Histological examination of the fracture side remains to be more accurate to discriminate between anatomical variants and fractures and to determine whether the fracture occurred during life (hemorrhages as "vital sign") or after death (Fig. 2).

\section{Fractures in perspective}

Previous studies on fatal pressure on the neck frequently focused on one specific event that led to death, for example, hanging and strangulation [40-43]. In hanging, at least one laryngeal fracture is reported in ranges starting from $9.5 \%$, $23.4 \%$ to $68 \%$ of the cases and less frequently also two or three larynx fractures, however, the amount of cases evaluated per study differed significantly between studies [41, 43-48]. The cricoid cartilage is usually not fractured by hanging; therefore, if a fractured cricoid cartilage is found, this could also indicate a homicide [44]. However, fractured cricoid cartilages are also extremely rare; in our study, we could only identify this in one case. Fractures of the thyroid cartilage 
were found to happen most frequently in cases of hanging [45]. The frequency of hyoid fractures in fatal strangulation cases is $55 \%$ with one or more fractures of the thyroid observed [49]. This percentage is more than twice as high as our results of the hyoid fractures. This may indicate that strangulation leads more frequently to hyoid fractures than our percentage suggests. The broad range of causes of death by fatal trauma on the neck, which were included in our study, can be the explanation. Compared to most studies about HLC fractures, our study had a high number of cases (284) evaluated. The great diversity of the causes of death in our cases makes a reliable incidence of HLC fractures difficult to gauge. Further studies are necessary to evaluate and combine fracture locations in HLC with the type of exerted violence on the neck.

\section{Postmortem radiology}

Noninvasive techniques that allow for assessment of osseous structures and soft tissue structures, such as CT scan and MRI scan, respectively, have proven their value to the postmortem process of analyzing the circumstances/cause of death. There is still a role for conventional X-rays in cases where the autopsy shows strong suspicion of violence on the neck and the pathologist removes the HLC for additional radiology (Fig. 2).

\section{Conclusion}

Fatal trauma on the neck leads most frequently to fractures of the superior horn of the thyroid cartilage and second most to fractures in the greater horn of the hyoid bone. Joints between the greater horn and body are frequently present, and dislocations in this joint are rare. If the explanted HLC is radiologically evaluated in a forensic setting, common and uncommon sites of fractures, the presence of joints, and dislocations should be examined thoroughly to gain as much information as possible for the pathologist in the context of further forensic investigation of the explant.

Acknowledgments We thank the pathologists, pathology assistants, and management from the NFI and the radiologists and forensic radiographers from the $\mathrm{GHH}$ for their continuous professional and pleasant cooperation.

\section{Compliance with ethical standards}

Conflict of interest The authors declare that they have no conflict of interest.

Ethical approval The used forensic database contained completely anonymized patient data, as previously described [28]. Institutional review boards of the GHH and NFI agreed to the collection and scientific use of data. According to the Dutch law, review by an ethical committee for research on anonymous retrospective data is not required.

\section{References}

1. Pollanen MS, Ubelaker DH (1997) Forensic significance of the polymorphism of hyoid bone shape. J Forensic Sci 42(5):890-892

2. Ubelaker DH (1992) Hyoid fracture and strangulation. J Forensic Sci 37(5):1216-1222

3. Rogde S, Hougen HP, Poulsen K (2001) Asphyxial homicide in two Scandinavian capitals. Am J Forensic Med Pathol 22(2):128-133

4. Cros J, Alvarez JC, Sbidian E, Charlier P, Lorin de la Grandmaison G (2012) Homicidal deaths in the Western suburbs of Paris: a 15year-study. Am J Forensic Med Pathol 33(4):404-409

5. DiMaio VJ (2000) Homicidal asphyxia. Am J Forensic Med Pathol 21(1):1-4

6. Saukko PJ, Knight B (2016) Knight's Forensic Pathology. CRC Press

7. Whyte AM (1985) Fracture of the hyoid bone associated with a mandibular fracture. J Oral Maxillofac Surg 43(10):805-807

8. Anthony R, Martin-Hirsch D, England J (2000) Dysphagia secondary to iatrogenic hyoid bone fracture. Br J Neurosurg 14(4): 337-338

9. Gupta R, Clarke DE, Wyer P (1995) Stress fracture of the hyoid bone caused by induced vomiting. Ann Emerg Med 26(4):518-521

10. Porr J, Laframboise M, Kazemi M (2012) Traumatic hyoid bone fracture - a case report and review of the literature. J Can Chiropr Assoc 56(4):269-274

11. Padgham ND (1988) Hyperextension fracture of the hyoid bone. $\mathrm{J}$ Laryngol Otol 102(11):1062-1063

12. Keerthi R, Quadri A (2016) Hyoid bone fracture: associated with head and neck trauma-a rare case report. J Maxillofac Oral Surg 15(Suppl 2):249-252

13. Roberts IS, Benamore RE, Benbow EW, Lee SH, Harris JN, Jackson A, Mallett S, Patankar T, Peebles C, Roobottom C, Traill ZC (2012) Post-mortem imaging as an alternative to autopsy in the diagnosis of adult deaths: a validation study. Lancet 379(9811): 136-142

14. Persson A, Lindblom M, Jackowski C (2011) A state-of-the-art pipeline for postmortem CT and MRI visualization: from data acquisition to interactive image interpretation at autopsy. Acta Radiol 52(5):522-536

15. Blaauwgeers J, van Rijn R (2012) Virtual autopsy-why not? Ned Tijdschr Geneeskd 156(19):A4786-A4786

16. Pollanen MS, Woodford N (2013) Virtual autopsy: time for a clinical trial. Forensic Sci Med Pathol 9(3):427-428

17. Takahashi N, Higuchi T, Shiotani M, Hirose Y, Shibuya H, Yamanouchi H, Hashidate H, Funayama K (2012) The effectiveness of postmortem multidetector computed tomography in the detection of fatal findings related to cause of non-traumatic death in the emergency department. Eur Radiol 22(1):152-160

18. Schwarzacher W (1928) Beiträge zum mechanismus des erhängungstodes. Dtsch Z Gesamte Gerichtl Med 11(1):145-153

19. Malek AM et al (2000) Patient presentation, angiographic features, and treatment of strangulation-induced bilateral dissection of the cervical internal carotid artery. Report of three cases. J Neurosurg 92(3):481-487

20. Schrag B, Vaucher P, Bollmann MD, Mangin P (2011) Death caused by cardioinhibitory reflex cardiac arrest-a systematic review of cases. Forensic Sci Int 207(1-3):77-83

21. Brouardel $P$ (1897) La pendaison, la strangulation, la suffocation, la mersion. Paris: J.-B. Baillière et fils. viii, $584 \mathrm{p}$

22. Moore LM, Persaud TVN (2008) The developing human: clinically oriented embryology, 8th edn. Saunders Elsevier, Philadelphia, pp $161-165$

23. de Bakker BS et al (2018) The development of the human hyoidlarynx complex revisited. Laryngoscope 128(8):1829-1834 
24. Miller KW, Walker PL, O'Halloran RL (1998) Age and sexrelated variation in hyoid bone morphology. J Forensic Sci 43(6):1138-1143

25. Kindschuh SC, Dupras TL, Cowgill LW (2012) Exploring ancestral variation of the hyoid. J Forensic Sci 57(1):41-46

26. Urbanová P, Hejna P, Zátopková L, Šafr M (2013) The morphology of human hyoid bone in relation to sex, age and body proportions. HOMO-J Comp Hum Biol 64(3):190-204

27. Pollard J et al (2011) Mechanisms of hyoid bone fracture after modelling: evaluation of anthropological criteria defining two relevant models. Forensic Sci Int 212(1-3):274 e1-274 e5

28. de Bakker HM, Soerdjbalie-Maikoe V, Kubat B, Maes A, de Bakker BS (2016) Forensic imaging in legal medicine in the Netherlands: Retrospective analysis of over 1700 cases in 15 years' experience. J Forensic Radiol Imaging 6:1-7

29. D'Souza DH, Harish SS, Kiran J (2010) Fusion in the hyoid bone: usefulness and implications. Med Sci Law 50(4):197-199

30. Gupta A et al (2008) Study of age of fusion of hyoid bone. Leg Med (Tokyo) 10(5):253-256

31. Pollanen MS, Chiasson DA (1996) Fracture of the hyoid bone in strangulation: comparison of fractured and unfractured hyoids from victims of strangulation. J Forensic Sci 41(1):110-113

32. Lebreton-Chakour C, Godio-Raboutet $\mathrm{Y}$, Torrents R, Chaumoitre K, Boval C, Bartoli C, Adalian P, Piercecchi-Marti MD, Thollon L (2013) Manual strangulation: experimental approach to the genesis of hyoid bone fractures. Forensic Sci Int 228(1-3):47-51

33. Dalati $\mathrm{T}$ (2005) Isolated hyoid bone fracture. Review of an unusual entity. Int J Oral Maxillofac Surg 34(4):449-452

34. Pollanen MS, Bulger B, Chiasson DA (1995) The location of hyoid fractures in strangulation revealed by xeroradiography. J Forensic Sci 40(2):303-305

35. Porrath S (1969) Roentgenologic considerations of the hyoid apparatus. Am J Roentgenol Radium Therapy, Nucl Med 105(1):63-73

36. Ahmad M, Madden R, Perez L (2005) Triticeous cartilage: prevalence on panoramic radiographs and diagnostic criteria. Oral Surg Oral Med Oral Pathol Oral Radiol Endod 99(2):225-230

37. Di Nunno N et al (2004) Anomalies and alterations of the hyoidlarynx complex in forensic radiographic studies. Am J Forensic Med Pathol 25(1):14-19
38. Ramchand $\mathrm{T}$ et al (2012) Management of hyoid bone fractures: a systematic review. Otolaryngol Head Neck Surg 147(2):204-208

39. Frederick C (1942) Textbook Surgery, Chapter: Fracture of the hyoid bone. Saunders, Philadelphia, p 146

40. Green H, James RA, Gilbert JD, Byard RW (2000) Fractures of the hyoid bone and laryngeal cartilages in suicidal hanging. J Clin Forensic Med 7(3):123-126

41. Luke JL, Reay DT, Eisele JW, Bonnell HJ (1985) Correlation of circumstances with pathological findings in asphyxial deaths by hanging: a prospective study of 61 cases from Seattle, WA. J Forensic Sci 30(4):1140-1147

42. Maxeiner H (1998) "hidden" laryngeal injuries in homicidal strangulation: how to detect and interpret these findings. J Forensic Sci 43(4):784-791

43. Betz P, Eisenmenger W (1996) Frequency of throat-skeleton fractures in hanging. Am J Forensic Med Pathol 17(3):191-193

44. Godin A, Kremer C, Sauvageau A (2012) Fracture of the cricoid as a potential pointer to homicide. A 6-year retrospective study of neck structures fractures in hanging victims. Am J Forensic Med Pathol 33(1):4-7

45. Nikolic S, Micic J, Atanasijevic T, Djokic V, Djonic D (2003) Analysis of neck injuries in hanging. Am J Forensic Med Pathol 24(2):179-182

46. Azmak D (2006) Asphyxial deaths: a retrospective study and review of the literature. Am J Forensic Med Pathol 27(2):134-144

47. Paparo GP, Siegel H (1984) Neck markings and fractures in suicidal hangings. Forensic Sci Int 24(1):27-35

48. Feigin G (1999) Frequency of neck organ fractures in hanging. Am J Forensic Med Pathol 20(2):128-130

49. Demirci S, Dogan KH, Erkol Z, Gunaydin G (2009) Ligature strangulation deaths in the province of Konya (Turkey). J Forensic Legal Med 16(5):248-252

Publisher's note Springer Nature remains neutral with regard to jurisdictional claims in published maps and institutional affiliations. 